\title{
Identification of Diethylene Glycol Monobutyl Ether as a Source of Contamination in an Ion Trap Mass Spectrometer
}

\author{
Christopher R. Gibson and Cheri M. Brown \\ Department of Drug Metabolism, Merck Research Laboratories, West Point, Pennsylvania, USA
}

\begin{abstract}
Tandem liquid chromatography-mass spectrometry coupled to online radioactive material detection (LC/RAM/MS/MS) is a technique that is used routinely for in vivo and in vitro drug metabolism studies and allows for a simultaneous correlation between radiochemical peaks and mass spectral data. The compound diethylene glycol monobutyl ether (DGBE), a component of a commercially available scintillation cocktail for RAM analysis, was identified as a source of overwhelming chemical noise in a mass spectrometer which was used in an LC/RAM/MS/MS configuration. In this report, we describe the identification of DGBE as the source of the chemical noise and the methods that were used to minimize the exposure of the mass spectrometer to volatile components of the scintillation cocktail. (J Am Soc Mass Spectrom 2003, 14, 1247-1249) (C 2003 American Society for Mass Spectrometry
\end{abstract}

$\mathrm{T}$ he use of liquid chromatography-mass spectrometry coupled to online radiochemical detection (LC/RAM/MS/MS) is a very important technique for many scientists, especially in the field of drug metabolism [1-3]. This technique allows for the profiling and identification of metabolites in biological matrices using the simultaneous acquisition of radiochemical and mass spectral data following administration of radiolabeled drug candidates to test species. The presence of high chemical noise in a mass spectrometer can severely compromise the performance of the instrument and has been the focus of several reports [4, 5]. In this report, we describe the identification and reduction of unwanted chemical noise observed in a LCQ Deca XP ion trap mass spectrometer that was coupled to an online radiochemical detector.

\section{Experimental}

\section{Reagents}

Acetonitrile, formic acid, and water were purchased from Fisher Scientific (Pittsburgh, PA). Diethylene glycol monobutyl ether (DGBE) was purchased from Aldrich (Milwaukee, WI). Ultima-Flo M scintillation cocktail was obtained from Packard Bioscience (Downers Grove, IL). All of the reagents used were high-pressure liquid chromatography (HPLC) grade or better.

Published online August 26, 2003

Address reprint requests to Dr. C. R. Gibson, Merck Research Laboratories, Merck and Co., Inc., WP75A-203, P.O. Box 4, West Point, PA 19486, USA. E-mail: christopher_gibson@merck.com

\section{Instrumentation}

Experiments were conducted using a ThermoFinnigan (San Jose, CA) Surveyor HPLC system coupled to both a ThermoFinnigan LCQ Deca XP ion trap mass spectrometer and a Packard Series 500TR online radiometric detector, allowing for simultaneous mass spectrometric and radiochemical detection (Figure 1). The ion trap mass spectrometer was operated using positive electrospray ionization (ESI) and the source parameters were as follows: The capillary temperature was $280{ }^{\circ} \mathrm{C}$, the capillary voltage was $22 \mathrm{~V}$, and the ESI voltage was maintained at approximately $4.5 \mathrm{kV}$. Nitrogen was used for both the sheath gas (50 arbitrary units) and auxiliary gas (5 arbitrary units). The mobile phase was a mixture (95:5) of $0.1 \%$ aqueous formic acid and acetonitrile containing $0.1 \%$ formic acid flowing at $120 \mu \mathrm{L} / \mathrm{min}$ directly into the ESI source. Collision-induced dissociation (CID) experiments were performed on the chemical noise ion and the collision energy was optimized for each experiment.

Additional experiments were conducted using an API 2000 triple-stage quadrupole mass spectrometer (Applied Biosystems, Foster City, CA) operated in the positive turbo-ionspray mode. The ionspray source parameters were as follows: The source temperature was $380^{\circ} \mathrm{C}$, the ionspray voltage was $4.5 \mathrm{kV}$, and nitrogen was used for the curtain, collision and source gases. A sample was prepared by diluting DGBE in mobile phase (same as above) and product ion spectra (MS/MS) were acquired by infusing the solution into the ionspray source at a rate of $15 \mu \mathrm{L} / \mathrm{min}$. 

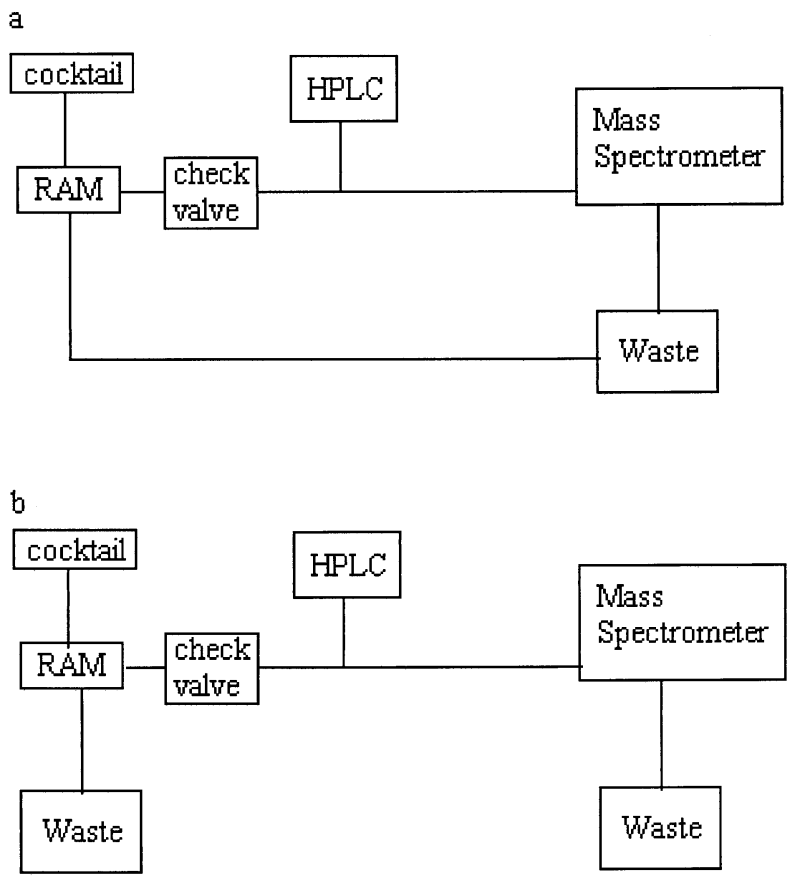

Figure 1. Configuration for tandem LC/RAM/MS/MS analysis. (a) The HPLC flow was split to the radiochemical detector (RAM) and mass spectrometer and the waste lines from each instrument were emptied into a single waste container. (b) The waste lines from the radiochemical detector and mass spectrometer were diverted into different waste containers to avoid contamination of the mass spectrometer source.

\section{Results and Discussion}

The tandem LC/RAM/MS/MS technique is routinely used in drug metabolism for metabolite identification and profiling of radiolabeled drug candidates and allows for a simultaneous correlation between radiochemical peaks and mass spectral data. However, in this case the technique created a problem by causing an overwhelming amount of interfering chemical noise in the mass spectrometer. The presence of overwhelming chemical noise directly impacts the quality of data derived from an ion trap mass spectrometer since the amount of ions that are injected into the trap for each scan is regulated in order to prevent space charging [6, 7]. Accordingly, a larger number of background ions means that fewer analyte ions are able to enter the trap, leading to lower sensitivity when acquiring full scan MS data. Since the metabolites requiring characterization are often present in the biological samples at low concentrations, sensitivity is critical for quality data.

Initial attempts to clean the ESI source with various solvents were largely unsuccessful, leading to a need to identify the origin of the chemical noise. Multiple lots of reagents and mobile phases were used and the chemical noise remained unabated. The reagents also were tested on other ion trap and triple-quadrupole mass spectrometers and the chemical noise ion at $\mathrm{m} / \mathrm{z} 325$ was not observed on those instruments. This indicated that the reagents that were used to compose the mobile phases

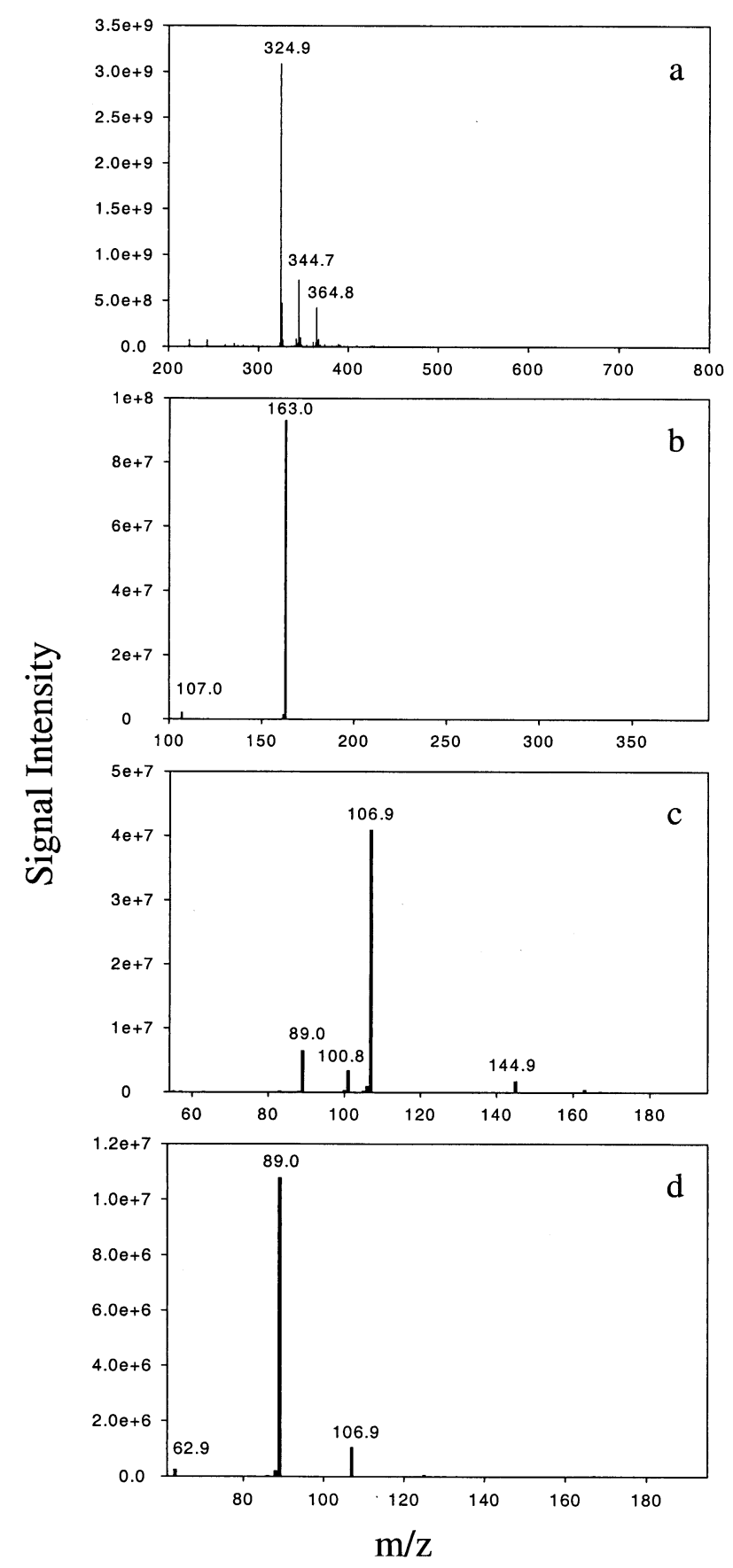

Figure 2. $\mathrm{MS}^{\mathrm{n}}$ spectrum of the chemical noise. (a) Full scan MS spectrum, (b) $\mathrm{MS}^{2}$ spectrum of $\mathrm{m} / \mathrm{z} 325$, (c) $\mathrm{MS}^{3}$ spectrum of $\mathrm{m} / \mathrm{z}$ 163, (d) $\mathrm{MS}^{4}$ spectrum of $m / z 107$. All mass spectra were acquired using a ThermoFinnigan LCQ Deca XP.

for LC/RAM/MS/MS analysis were not the source of the chemical noise. As can be seen in Figure $2 \mathrm{a}$, the chemical noise in the full scan mode had an intensity of approximately $3 \times 10^{9}$, which was significant enough to interfere with analytes of low concentrations. From the MS $^{2}$ scan (Figure 2b), it became apparent that the $\mathrm{m} / \mathrm{z}$ 325 ion probably was a proton-bound dimer of a compound with a molecular weight of 162 daltons. The $\mathrm{MS}^{3}$ and $\mathrm{MS}^{4}$ spectra (Figure 2c, d) each contained, 


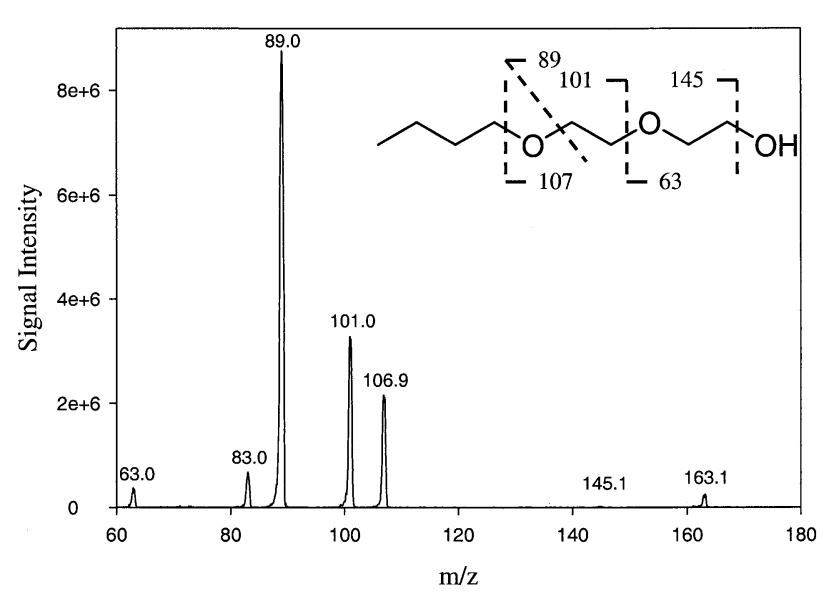

Figure 3. Product ion spectrum of DGBE using an API 2000 triple-stage quadrupole mass spectrometer with proposed fragmentation pattern.

among other fragments, a water loss (18 daltons) which was potentially indicative of a hydroxylated molecule.

With the knowledge of the apparent molecular weight of the chemical noise, a search of the CRC [8] was performed to identify molecules that had a molecular weight of 162 daltons. The lengthy list was initially narrowed down based on the isotope pattern of the chemical noise ion such that any molecules with distinct isotope patterns, such as halogen containing molecules, were eliminated from consideration. The putative CID fragments of the remaining molecules were predicted to find one that may have yielded the CID spectra of the chemical noise. The compound that appeared to be the best match for the CID data of the chemical noise was DGBE, which coincidentally was a component of the Ultima-Flo M scintillation cocktail used for the online radiochemical detector, accounting for approximately $10-14 \%$ of the cocktail volume [9]. To confirm the identity of the chemical interference, DGBE standard was obtained and a dilute solution of it was analyzed on an API 2000. The product ions of DGBE that were formed using the API 2000 (average of 10 scans) were consistent with those formed by the chemical noise on the Deca XP, thus confirming the identity of the interference (Figure 3).

Once the interference molecule had been identified, it was necessary to determine how it had contaminated the mass spectrometer source. Under normal operating conditions, the HPLC flow was split post-column such that it passed to the radiochemical detector and the mass spectrometer simultaneously (Figure 1a). A check valve was installed between the splitter and the radiochemical detector to prevent the back-flow of scintillation cocktail from the radiochemical detector to the ESI source. The system had been configured such that the waste from the radiochemical detector, containing a 3:1 ratio of scintillation cocktail to column effluent, and the gas exhaust lines from the ESI source were flowing into the same waste container (Figure 1a). The DGBE appar- ently had migrated up the gas exhaust lines from the waste container and accumulated in the mass spectrometer source. In order to reduce the interference, the waste lines from the radiochemical detector and the mass spectrometer were separated such that they flowed into separate containers (Figure $1 \mathrm{~b}$ ) and all of the lines, including the waste and gas exhaust, were replaced. Finally, the ESI source and atmospheric pressure ionization stack both were disassembled and cleaned with (1:1) methanol:water followed by acetone to reduce any DGBE contamination. Following these reorganizational and maintenance procedures, the chemical noise produced from DGBE was significantly reduced to a level that allowed for normal mass spectrometer function. This example highlights the importance of a clean ESI source for proper mass spectrometer function and how complementary technologies can compromise the integrity of mass spectral data when appropriate precautions are not taken. Although this chemical noise was observed using an ion trap mass spectrometer, it should be noted that this type of interference may potentially be a problem on other mass spectrometers (i.e., triple-quadrupoles) which are used in an LC/RAM/MS/MS configuration.

\section{Acknowledgments}

The authors thank Dr. Bart Emary (Preclinical Drug Metabolism, Merck Research Laboratories, West Point, PA) for his suggestions during the preparation of this report.

\section{References}

1. Dalvie, D. Recent Advances in the Applications of Radioisotopes in Drug Metabolism, Toxicology and Pharmacokinetics. Curr. Pharm. Design 2000, 6, 1009-1028.

2. Dain, J. G.; Collins, J. M.; Robinson, W. T. A Regulatory and Industrial Perspective on the Use of Carbon-14 and Tritium Isotopes in Human ADME Studies. Pharm. Res. 1994, 11, 925-928.

3. Inskeep, P. B.; Day, W. W. Preclinical Drug Metabolism Studies and Strategies. Handbook of Drug Metabolism; In Woolf, T. F., Ed.; Marcel Dekker, Inc.: New York, NY, 1999; p 551.

4. Verge, K. M.; Agnes, G. R. Plasticizer Contamination from Vacuum System O-Rings in a Quadrupole Ion Trap Mass Spectrometer. J. Am. Soc. Mass Spectrom. 2002, 13, 901-905.

5. Aebi, B.; Henion, J. D. The Reduction of Chemical Noise in an Atmospheric Pressure Ionization/Ionspray Interface for Mass Spectrometry. Rapid Commun. Mass Spectrom. 1996, 10, 947-951.

6. Bier, M. E.; Schwartz, J. C. Electrospray-Ionization Quadrupole Ion-Trap Mass Spectrometry. Electrospray Ionization Mass Spectrometry; In Cole, R. B., Ed.; John Wiley and Sons, Inc.: New York, NY, 1997; pp 243-244.

7. Cox, K. A.; Cleven, C. D.; Cooks, R. G. Mass Shifts and Local Space Charge Effects Observed in the Quadrupole Ion Trap at Higher Resolution. Int. J. Mass Spectrom. Ion Processes 1995, 144, 47-65.

8. CRC Handbook of Chemistry and Physics (3rd Electronic Ed.); http://www.knovel.com/knovel2/Toc.jsp?BookID=34 (Accessed Dec. 2002).

9. Ultima-Flo Material Safety Data Sheet, Packard Bioscience Company, 1999. 